\title{
Multiscale Analysis of Activity Rhythms of Animals
}

\author{
B. M. Wu ${ }^{1}$, F. K. Lam ${ }^{1}$, Francis H.Y. Chan ${ }^{1}$, Paul W. F. Poon ${ }^{3}$ and A. M. S. Poon ${ }^{2}$ \\ ${ }^{1}$ Department of Electrical and Electronic Engineering, University of Hong Kong, Hong Kong \\ 2 Department of Physiology, University of Hong Kong, Hong Kong \\ ${ }^{3}$ Department of Physiology, School of Medicine, National Cheng Kung University, Tainan, Taiwan
}

\begin{abstract}
In this paper we propose a multiscale approach to analyze locomotion activity rhythms of animals. The Wavelet Transform (WT) is used to decompose the locomotion signal into different scales. An activity-section segmentation procedure is introduced to segment different scale activity sections following a strategy of from large scale to small scale with the results arranged in a tree description. Furthermore, each activity segment can be characterized by several parameters. Initial study results on mice are presented.
\end{abstract}

Keywords -- Wavelet transformation, Multiscale decomposition, Activity rhythms, Mice

\section{INTRODUCTION}

Quantitative assessment of activity rhythms is desirable in behavioral studies of humans or animals, and the Fourier spectral analysis has been used to extract activity rhythms [1]. But the method suffers from poor time resolution because its amplitude-frequency spectrum does not have temporal information related to the activity events. In reality, there are numerous rhythms encountered in biology and medicine and their periods may range from a fraction of a second to a few hours or even several weeks [2]. Furthermore, some of them may be adjusted from time to time or change with respect to environments. The short-term periodic variations (as high frequency components) are superimposed upon the long-term activation (lower frequencies) such as circadian activation. There is a clear need for effective methods which would extract the rhythms and track their variations.

In consideration of above, a multiscale approach has been proposed by using the Wavelet Transform (WT) which is a temporal dynamic spectral analysis and has good localizing ability in both time and frequency fields. In the frequency field it is equivalent to a series of filter banks decomposing a complicated signal into multiple sub-band components. All the above make it useful to become a good alternative to FFT. To confirm that an initial study has been carried out.

\section{Data Collection}

In order to extract both long-term and short-term activities, the locomotion of the animal in a cage is recorded. An image processing-based locomotion acquisition system has been developed [3]. A dynamic background tracking based image thresholding method was used to take into account of ambient lighting variation. At every second, one frame was digitized with 8-bit per pixel resolution. Difference images between successive frames were obtained as displacement area which form the index for the animal movement activities. Experiments were carried out on individual mouse under an artificial Day (D) and Night (N) cycle (Fig. 1(a)). Each time the locomotion data of one mouse over three days were collected. In this activity rhythm study, a relatively low temporal resolution was chosen, so a coarse resolution locomotion signal, (the root mean square value $L_{R M S}$ over a moving time window as shown in Fig. 1(b)), was computed from the original data set $L(t)$. Fig. 1(b) shows a profile of $L_{R M S}$ over three days with time window $\mathrm{T}=7.2$ minutes.

$$
L_{R M S}=\sqrt{\int_{f}^{T} L^{2}(t) d t}
$$

\section{Wavelet Decomposition}

The WT was computed by the iterative method of Mallet [4], which yields wavelet coefficients at each level in a hierarchical way, and at each successive level the signal resolution is decreased by a factor of 2 . In our study six scales were used, and the $L_{R M S}$ locomotion signal $A_{0}$ was decomposed into six approximated signals of $A_{-1}$ to $A_{-6}$, together with six detail signals of $D_{-1}$ to $D_{-6}$. Fig. $1(c)-(n)$ give their normalized and interpolated plotting. The approximated and detail signals at each scale were subjected to statistical analysis.

\section{Statistical Analysis}

From the decomposed approximated and detailed locomotion signals several parameters were extracted.

i) The energy in each sub-band was extracted by calculating the RMS of the detailed signal which provides an overall indicative of a specific characteristic of rhythm.

ii) The locomotion signals in each scale were further characterized by four parameters: the number of activity 
sections and their borders, centers and energies. A strategy of from coarse to fine scale was adopted. We segmented the activity sections starting from the largest scale approximated signals $\mathrm{A}_{-6}$ by searching the valleys (down arrow of Fig. 1(n)) and peaks (up arrow of Fig. 1(n)). The valleys were defined as borders between two adjacent activity sections and the peaks as activity centers. The energy in each activity section can be computed.

iii) Finer movement patterns can be obtained by the same procedure by analyzing from $A_{-5}$ down to $A_{-1}$ traces to review more detailed activity sections. By this method, we can successfully search the features starting from the slow to fast varying features. The parameters obtained can be conveniently arranged as data tree structure.

From the set of results shown in Fig. 1, we can interpret the $A_{-6}$ of Fig. 1(n) as giving three activity sections which correspond to the circadian rhythm. From the fourth scale $A_{-4}$ as shown in Fig. 1 (1), within the first two sections in the A-6 trace we can see two small activity sub-sections. In the $A_{-6}$ third section, there are three sub-sections in $A_{-4}$. This process can be extended to the finer resolution traces. From the figure, we noticed the parameters are quite repeatable over three-day period.

\section{Conclusion AND Discussion}

Initial study suggests the multiscale approach is able to extract the activity components with different temporal scales. The multiscale decomposition and the tree description provide a muti-resolution viewing on the activity of animals which allows us to find out in which scale the activity rhythms or patterns are changed, and then have a better understanding on activity patterns. This new detection method is a first attempt using wavelet to analyze the activity rhythms.

\section{ACKNOWLEDGES}

Supported in part by grants from CRCG, RGC and BRI. We also thank Peter Ng and Y. K. Pang for their technical assistance.

\section{REFERENCES}

[1] J. Beau, " Activity Rhythms in Mice III: Stability and Plasticity of Rhythm Characteristics in Experimental and Environmental Conditions", Physiology \& Behavior, vol. 52, pp. 231-235, 1992.

[2] E. Haus and Y. Touitou, "Principles of Clinical Chronobiology", Biological Rhythms in Clinical and Laboratory Medicine, Springer-Verlag, Berlin, 1992.

[3] B. M. Wu et. al., "A Microcomputer-based system for Long Term Analysis of Activity Profiles in Rats", Proc.
Hong Kong Biomed. Engin. Conf. BME'94 2: 355-358, 1994

[4] S. G. Mallet, "A Theory of Multiresolution Signal Decomposition: the Wavelet Representation", IEEE Trans. Patt. Anal. Machine Intell., Vol. 11, pp. 674-693, 1989.

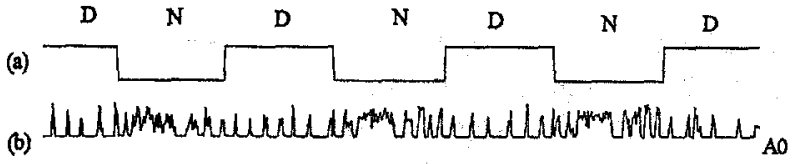

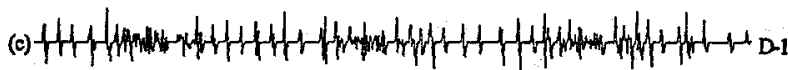

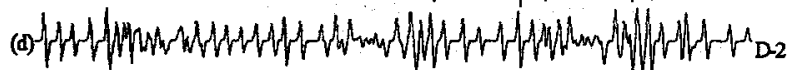

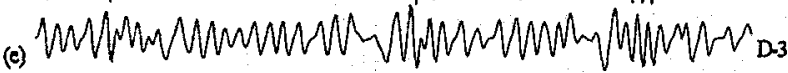

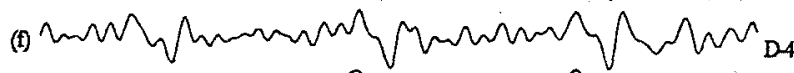
(g)
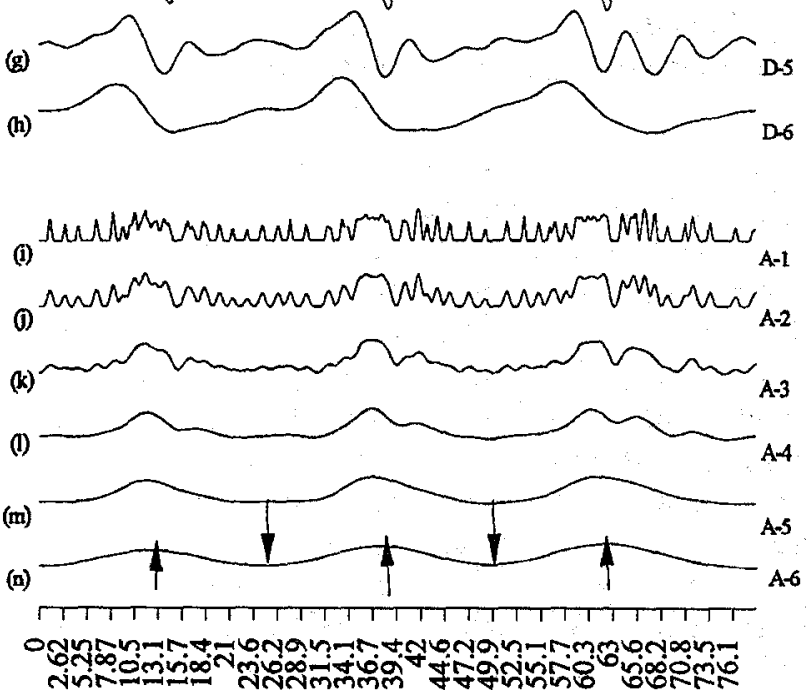

Time(Hours)

Fig. 1 Multiscale decomposition of the locomotion signal of one mouse over three days. (a) is the day and night cycle, (b) is the $L_{\text {RMS }}$ computed from the raw data, (c)-(h) are decomposed detailed signals and (i)-(n) are decomposed approximated signal. 